\title{
Diagnosis of Ailment in Gaseous Matrix (Air) at Operation Suite - A Detailed Insight
}

\author{
Aswin Sidhaarth K. R. ${ }^{1}$, Shabarish. S ${ }^{2}$, Vinod Kumar. $\mathbf{M}^{3}$, Tamilarasan. $\mathbf{K}^{4}$ \\ ${ }^{1,3}$ Associate Professor, Department of Civil Engineering, Vel Tech Rangarajan Dr. Sagunthala R\&D Institute of \\ Science and Technology Avadi, Chennai -62. \\ ${ }^{2}$ M.Tech Environmental Engineering II Year Student, Vel Tech Rangarajan Dr. Sagunthala R\&D Institute of \\ Science and Technology. \\ ${ }^{4}$ Assistant Professor, Department of Civil Engineering, Vel Tech Rangarajan Dr. Sagunthala R\&D Institute of \\ Science and Technology, Avadi, Chennai -62.
}

aswincivil@gmail.com ${ }^{1}$, shankaranshabarish1997gmail.com ${ }^{2}$, m.vinodkumar.civil@gmail.com ${ }^{3}$, tamilkaruppiah@gmail.com ${ }^{4}$

Article History: Received: 10 November 2020; Revised: 12 January 2021; Accepted: 27 January 2021;

Published online: 05 April 2021

\begin{abstract}
Operation suite in hospitals is an environment where airborne microorganisms spread quickly. In operation suites airborne microorganisms spread through wounds of the patients, surgical instruments and gloves, air conditioner etc. Various health effects such as, gasping irritation and nonspecific symptoms, inhaling infections, asthma and allergy, Bagassosis and organic dust toxic syndrome, and chronic obstructive pulmonary diseases are created by these airborne micro organisms. So in order to reduce or avoid this problem it is essential to monitor the quality of air in operation suites and also to reduce the effects of airborne microbes. This study aims to show the different methods which can be adopted to bring down the serious consequences of airborne microorganisms on the individuals who are in the operation suite.
\end{abstract}

Keywords: Air quality of operation suite, Airborne microorganisms, Health effects.

\section{Introduction}

\subsection{Quality of indoor air}

Quality of indoor air can be described as the quality of air which is present within a particular structure such as hospitals, schools, residential buildings. The quality of indoor air is usually polluted or distressed by chemicals including gases such as carbon monoxide, ozone, radon), volatile organic compounds (VOCs), particulate matter (PM) and fibers, organic and inorganic contaminants, and biological particles such as bacteria, fungi. (AlessandraCincinellietal,2017)2.

The primary reason for pollution of indoor air are: incineration, construction materials, and biosprinkler. The pollutants for indoor air are acaricides, heavy metals, radon, environmental tobacco smoke, asbestos, volatile organic matter in developed countries. In the nations which are developing, biomass fuels generated from combustion products contribute most to indoor air. (Ankita Kankariaetal ,2014)1.

The quality of indoor air in recent years has attained more recognition from the social groups, and environmental administration in order to take care the wellbeing of building occupants. So many observations regarding this subject have shown variations through the years in the quality of indoor air, with increase in pollutants and their levels, the health and life standard of several people were affected by poor indoor air quality. The health risks of indoor pollution is also greater than those associated with outdoor pollution. To be precised, poor quality of indoor air creates more serious and harmful consequences to children, young adults, the elderly, or those suffering chronic respiratory and or cardiovascular diseases (Alessandra Cincinelli et.al ,2017)2.

\subsection{Health effects caused by indoor air pollution}

Indoor air pollution will cause so many serious health effects, including migraine, inhaling issues, colds, painful (sore) throats, chronic cough, skin allergy, eye irritation, lethargy, dizziness and loss of memory. Longterm effects may include increased risk of cancer. Aged people and children are those who gets affected by vulnerable problems like asthma, allergies and heart and lung diseases (Jane E Brody 1981)27.

(Tharwat Mokalleda et.al 2019 )55, made a study on indoor air quality of Beirut-Rafic Hariri international airport (RHIA)-Lebanon. In this study using gas chromotographic technique 46 volatile organic compounds are assessed and using calorimetric technique, nitrogen dioxide concentration are measured and it is found that 
except acrolein volatile organic compound doesn't cause any risks and nitrogen dioxide concentrations are significantly below the world health organization (WHO) yearly threshold limit $(40 \mu \mathrm{gm}-3)$.

The national institute of environmental health and science shows percentage of health effects caused by the indoor air pollution in the figure 1 and the health impacts of poor air quality is shown in the figure 2 (Louisvilleky,2020)33.

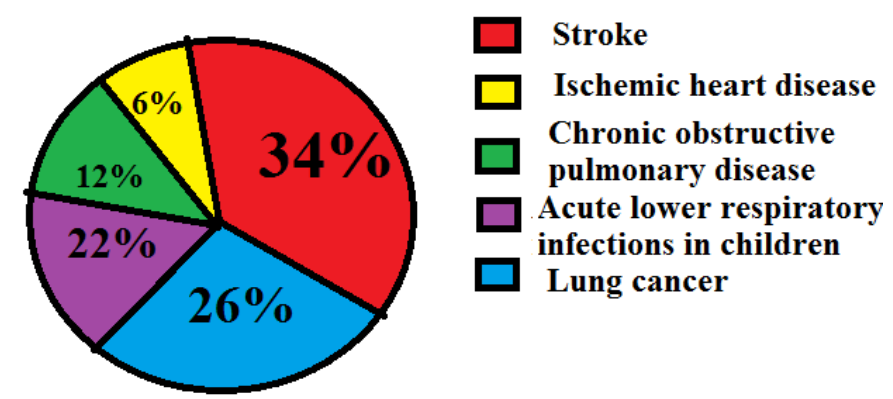

Figure 1. The national institute of environmental health and science shows the percentage of health effects caused by the indoor air pollution (Deepshika, 2018).

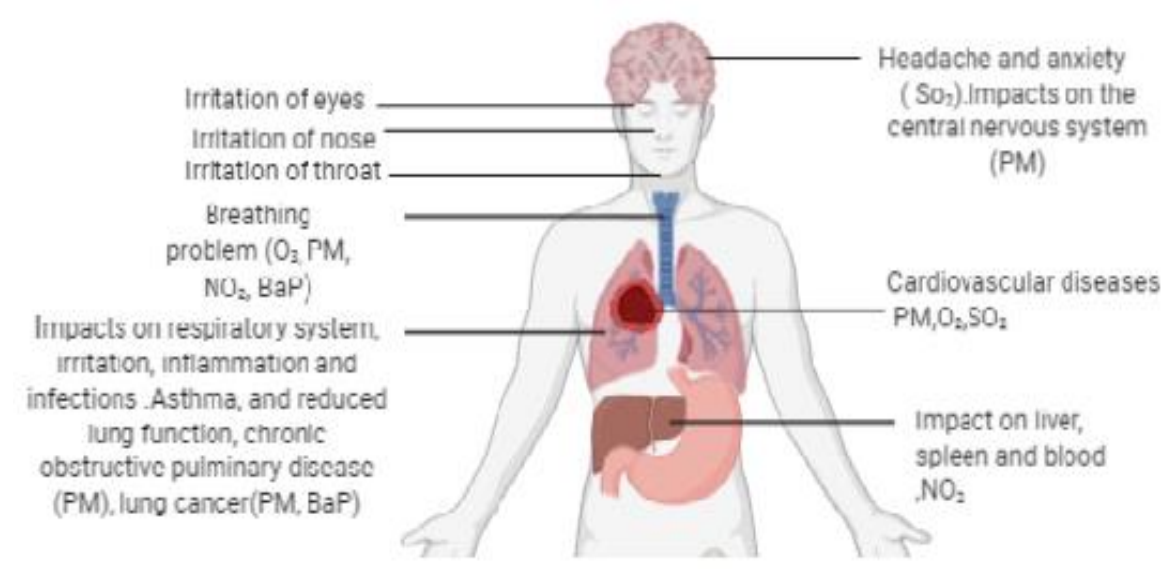

Figure 2. Health impacts of poor air quality in a human body

1.3. History behind the pollution of air in the operation suite of hospitals

The history of indoor air quality environment starts almost 1.5 million years ago when the early stages of people started to use campfires for cooking. When these campfires was bought into their houses such as huts there is a small opening on the roofs through which the smoke can escape. Right from the history people understood that the polluted air can cause health issues. In 1853 a professor from Germany Munich, in hygiene found that the 'bad indoor air doesn't make people sick but such air may decrease the resistance power of people against airborne microbial agents which can create illness (Lada Hensen Centnerová, 2018)37. Indoor air quality is one of the major environmental issues for more than hundred years since 1850's (ie) from the beginning of revolution on hygienic perspective (Sundell J, 2004)50.

Since so many centuries, the indoor air has been considered to be the major path way for transmission of infectious diseases. Since1950s the place for pathogens to live and grow for surgical site infections (SSIs) were thought to be the nasopharyngeal (The nasopharynx is the upper part of the pharynx (throat)behind the nose) region of the surgical team and the operating room air (Kappstein, 2001)30. During 1960s the seperated studies were made first time. Few intense surgical site infections occured after total hip or knee replacement operations when laminar airflow (LAF) ventilation was used. Various studies were published reporting this. (Lidwell et al, 1980)34. There is a reduction of infection rate in this study. After aliterature review was conducted by (Kappstein, 2001)30, he reported that the droplet nuclei which consist of airborne pathogens could only originate from the nasopharyngeal (upper part of the pharynx (throat) behind the nose region) and from the sacles of skin of surgical people. By using HVAC (Heating, ventilation and air conditioning) systems with turbulent mixed 
airflow, there is a possibility of spreading of bacteria from individuals at the boundary of the operating suite to the wound. Therefore, the supply of HVAC systems should be made throughout the surroundings of the surgical field and instrument table with air having a low microbial count (Lidwell et.al, 1980)34.

The principle of airflow would need to be stable laminar airflow (The movement of air in same speed and in same direction) ventilation (Kappstein, 2001)30. Operating rooms are difficult systems where such a big amount of risk factors favor the origin of infectious complications. There are so many parameters which decides the risk factors of operating room such as structural design of operation rooms, facilities available there, the patient condition, the sort of surgery which is completed to the patients, the behaviors of the surgical team, how often people enter and leave the operation rooms, and also the efficiencies of the (HVAC) systems. The danger of infection during hip surgery and knee arthroplasties (replacement of joints) increased from $1.99 \%$ to $2.18 \%$ and from $2.05 \%$ to $2.18 \%$ within the USA, between 2001 and 2009. By 2030, the chance of infection is predicted to extend to $6.5 \%$ and $6.8 \%$, respectively. The foremost recent arthroplasty yearly report of Italy stated that $18.9 \%$ of knee arthroplasty revisions and $7.8 \%$ of hip revisions were secondary to infectious complications. The material exposure in air causes bacterial and viral respiratory infections (Carmen Irniguez et.al, 2020$) 14$.

A association between contamination of airborne microbes and deep SSI (Surgical site infection) incidence in prosthetic joint surgery was shown once by a medical research council study where ultraclean operation theatres were recommended for this kind of surgery. The united kingdom national joint registry report says that, during 2003 -2006 period, infection was liable for about 19 acute renal failure of joint surgery. (Sandiford et.al ,2009)51. Microbes within the particles in air stay over the laceration, dressings and surgical instruments and cause septicaemia (Chow et.al ,2005)11.

(Whyte et.al, 1982)57 found that contamination from skin of the patient will cause infection in 2\% cases and from operation suite people in $98 \%$ cases. They also found that in $30 \%$ cases, contaminants reach the wound from operation suite individual via air and in $70 \%$ cases it is through hands. A study has been carried out based on preserving the indoor air quality in operation suites. Various parameters such as temperature of air, humidity level, carbon dioxide (CO2) particulate matter (PM), and bacterial concentrations were monitored and noted. At the end it was found that the indoor air quality varies from month to month. Gram positive bacteria was found and the COD level in the operation rooms were below the suggested level (600 ppm average over 8 hours) set by Taiwan's environmental protection agency.

Dust particles act as a medium for transporting microbes which can cause infection in wounds (Neil 2005 )46. Many countries throughout the globe have recognized the issue of air quality in operating rooms. The guidelines of Nordic countries are most sternest in managing surgical smoke.

Countries like London and Canada have documents for guidance on the consequences of smoke which occurs in every surgery. A number of international associations like the operating room nurses and also the American society for laser medicine and surgery have produced strong statements that recommends the application of local exhaust ventilation. Air quality could be a measure of how clean or polluted the air is. Airborne microbes as airborne toxins passed from one victim to the subsequent through the air, without physical contact, causing very least irritations. Surgical site infections can even be there after operations due to airborne microbes. Surgical site infections are related to substantial morbidity (a condition during which a patient suffers from a disease) and mortality (death). However, mortality also occurs in hospitals due to climatic changes (temperature changes) (Carmen Iniguez et.al, 2020 )16.

Surgery interventions are one in every possible way for infections in operating suites. Patients who have low immunity power are immediately affected by infections that are located on their wound after surgical activity. Contamination within the surgical field occurs from various sources like (direct way) human contamination and (indirect way) through instruments, gloves, and components. However, both direct and indirect contamination rely on indoor air quality of operation room and ventilating system, also on medical team behaviors, patients, health, surgical procedures. An ideal indoor air quality inside the operating room can prevent the amount of surgical site infections by controlling the amount of total airborne contamination. Air is considered to be a vector of pathogens and also a very important reason for surgical site infections (SSI). Some surgical site infections can be superficial involving the skin only, whereas some others can be more serious and involve deeper tissues under the skin organs. Surgical site infections represent the most common healthcareassociated infections among patients, constituting up to $19.6 \%$ of all such infections in Europe in 2011-2012 (J.M. Badia etal ,2017)8. In the US, a survey based on healthcare-associated infections says that 157,500 surgical site infections occured with surgeries of inpatients in 2011. In Sweden, among 65,000 patients who suffer from 
healthcare related infections each year, $23 \%$ of them are surgical site infected persons (S.S. Magill et.al, 2014)44.

In order to control or reduce the airborne microbes contamination in operation theaters, the air in the operation theaters should be made as much as pure with less pollutants.

\section{Challenges in Purification of Indoor Air}

Indoor air quality (IAQ) can be defined as the standard of air within buildings, especially because it is associated to the health and luxury of building individuals. Indoor air quality will be plagued by gases, like carbon monoxide gas or radon; microbial contaminants, like bacteria because of moisture intrusion; improper and inadequate ventilation or make-up air; or the other pollutant or condition that may cause adverse health conditions. The U.S. Environmental Protection Agency (EPA) says that individuals who is also exposed to indoor air pollutants for the longest periods of time are often those most at risk of the consequences of indoor pollution. Such groups include the young, the elderly, and therefore the chronically ill. Air quality and air purification has become an enormous challenge (Bryan Heitzmann, 2015$) 7$.

In operation suites, purifiers like filters may noticeably reduce concentrations of small harmful particles. Here the upkeep costs of filters are possibly larger. Majority of air purifiers use cellulose and membranes of polymer that are changed each month roughly, often as a part of a daily contract. The air is made to pass through the filters with fans and pumps which consume energy, which is equivalent to a bright light bulb ie 100 watt. ( Alastair Lewis, 2019)3. A filter may find holding milligrams (and is also more) of individual chemicals that were initially found in air at very diluted concentrations, and whose previous fate was probably to deposit as a really thin layer over huge areas of land when the filters become repair or of no use (Alastair Lewis, 2019)3.

Photo catalysis that utilizes semiconductor nano particles may be a comprehensive technology for the purification of air. Although photo catalysis investigation is completley analysed, it still falls under the need of practical usage (ie) lack of effective actinic radiation activity to utilize ambient light and catalyst deactivation

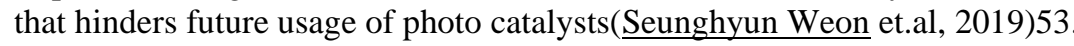

Air purifiers that eliminates dust aren't good in getting obviate bad odor and contrariwise. There are few air purifiers that release large amount of ions within the air and also ozone gas may get released which will be harmful. It is very difficult for such appliances to free the air from gases and odors (Alastair Lewis ,2019)3.

The surgical instruments are one amongst the main sources through which airborne microbes gets spread which ends up in surgical site infections (D chauveux, 2015)13, so it's essential to sanitize or clean the surgical instruments after each surgery. This may not be done at sometimes because of lack of concentration or tension during the surgery or if there's not much available time for the subsequent surgery (ie) in case of any emergency.

\section{Various Methods to Reduce Air Pollution in Operation Suite}

\subsection{Increase in supply of air}

In 1964 Charnely found when a joint replacement surgery was done in an operating room the airborne microbes causes infection in wounds of the patient. So as a solution to this problem, he decided to extend the availability of air and reduce the concentration of airborne microbes. In an operating room a clean air enclosure system was kept and the activity was conducted. In this the provision of air supply is increased ie (300 air changes/ hour) and as a result of this contamination of airborne microbes is reduced and therefore the surgical site infection got decreased. Clinical trials were administered in Britain, Europe, and in US and after these trials it's been proved that between $80-90 \%$ of bacterial contaminants found within the wound after surgery came from colony forming units (cfu) present within the operating room air. When the numbers of colony forming units gets decreased, there's a corresponding reduction within sepsis of wound. The sources, concentration, and movement of airborne cfu during a surgery are found, and various ways of reducing their numbers are analysed. Only reduction of cfu within the operating zone less than $1 / \mathrm{m} 3$, by means of an exponentially curved flow of microbiologically clean air, along with a complete body exhaust worn by the members of the surgical team and also the patient, provides aseptic conditions without imposing restrictions or changes in surgical operation (AP Spagnalo ,2013)52. 


\subsection{Ventilation Systems}

In Operating suites the aeration is important to control airborne contamination and to decrese the risks of surgical site infections (SSIs) (AP Spagnalo, 2013)52. The four categories of operation theatre ventilation system are here listed:

- A-Partial Uni Directional Air Flow (P-UDAF) — designed (airflow rate and ceiling area) to achieve an ISO 5 air cleanliness class in "at-rest" condition.

- B-Partial Uni Directional Air Flow (P-UDAF) - designed (airflow rate and ceiling area) to achieve an ISO 7 air cleanliness class in "at-rest" condition.

- C-Mixing Air Flow (MAF) — designed to achieve an ISO 7 air cleanliness class in "at-rest" condition and adopting high-wall supply grilles.

- D-Mixing Air Flow (MAF) — designed to ISO 7 air cleanliness in "at-rest" condition and adopting ceiling air diffusers.

Since 1940s infection in wounds through contamination of airborne microbes has been studied together with the analysis of operating suites with differing kinds of ventilation systems, on the effect on transfer of bacteria from one place to another, and on possibilities of airborne reduction. Regarding these studies, two main sorts of ventilation systems for operating theatre environments are identified (ie) mixing ventilation airflow (MAF) and partial unidirectional airflow (P-UDAF). The blending system dilutes airborne contamination by turbulent mixing between supplied clean sterile air and contaminated room air. At the identical time, the partial unidirectional air flow system, because of its low turbulence flow that is generally directed downwards, moves the contaminants and finally it is removed from the operating room protected area (around the surgical table) and reduces the danger of cross-contamination (transfer of bacteria from one place to another) from the outer zone where airflow conditions aren't unidirectional. The blending air flow (MAF) system with terminal mixing diffusers and therefore the partial unidirectional air flow (P-UDAF) system with unidirectional ceiling systems supply sterile air are cleaned by high efficiency HEPA air filters (Melhado et.al ,2006)39. Figure 3 shows the image of ventilation performance operational suites.

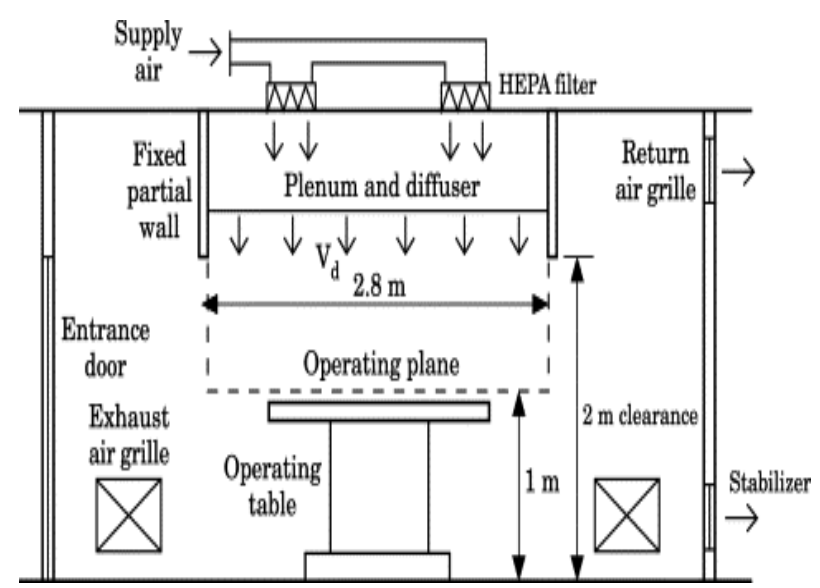

Figure 3. Ventilation performance in operation suites

Surgical site infection risk due to airborne bacteria is the main reason for developing ventilation design and monitoring procedures in operating theatre.

Ventilation is mandatory in all occupied premises especially in operating rooms. Several factors such as fresh air supply for human habitation, control of airborne bacteria, thermal comfort, humidity level determine the requirements of ventilation systems. For the function and efficiency of an operating rooms, the ventilation system is considered to be the main factor. It controls the level of the airborne contaminant, which often presents post-operative wound infection (surgical site infections) risks to the patients. Hence, the designing and planning of ventilation systems in operating rooms are crucial so that both infection rates and operating costs are lowered. The mechanical system is crucial for infection control within the operating room, i.e. to safeguard the patient from becoming infected and also for keeping good indoor air quality (IAQ). In accordance with the indoor air quality of an area which is set by the amount of indoor air pollution and other characteristics that includes the air temperature, ratio and air speed. Pollutants in an surgery include aerosols and anesthesia gases and a big consideration is that differing types of ventilation systems have different designs, dimensions, applicability and resultant airflow dynamics (Friberg et.al., 2003)22. In some cases the ventilating system can decrease the 
share of infection. Some styles of ventilation systems improve infection control, because they supply airflow dynamics that "divide" the hospital room in several zones, ensuring different protection levels. Figure 4 shows the three zones, zone A, zone B and zone $\mathrm{C}$ in line with the various varieties of ventilation systems provided. ( Melhado et.al, 2006)39

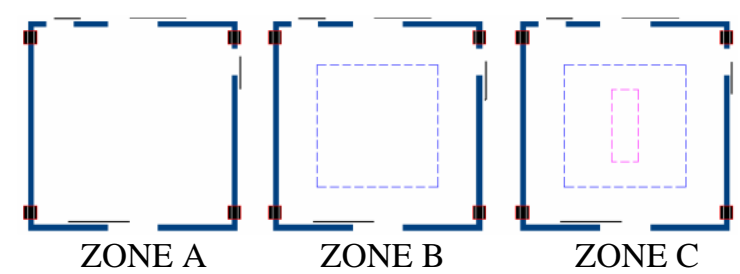

Figure 4. Different types of ventilation system according to the three zones

\subsubsection{Conventional Ventilation Systems}

In old hospitals conventional (mixing) ventilation systems are employed in operating rooms. These sort of ventilation systems are generally used for general surgeries, as an example, abdominal Surgery. Out of the above mentioned three zones in the figure 4 only 1 zone is implemented within the operating room through dynamic turbulent airflow. The traditional ventilation isn't an improved option for the surgery, mainly in surgeries, because dynamic turbulence air flow doesn't provide clean air within the patient area and also on the instruments table. Different disposition on the ceiling and walls may occur by providing conventional ventilation systems (Melhado et.al, 2006)39.

\subsubsection{Plenum ventilation systems}

Plenum ventilation systems are the systems within which from a chamber usually called as a plenum chamber through mechanical process at a pressure greater than air pressure fresh or pure air is forced into a ventilated space so as to clear the foul air. The foremost common ventilation systems employed in operating rooms are plenum ventilation systems. In countries like Netherland Plenum ventilation systems are used. These ventilation systems are often used for various kinds of surgery. Various sorts of plenums utilized in European country are Big size-plenum (3.0mx3.0m), Plenum (1.2mx2.4m and 2.4mx2.4m), 3Tplenum and the orthogonal plenum. The $3 \mathrm{~T}$ plenums and plenums are used for newly built or to the renovated operating theatres, which have provided satisfactory leads to environmental control. The massive size-plenum divides the hospital room in two zones while the 3T-plenum divides the operatingsuite in three thermal zones and two zones of protection (Melhado et.al, 2006)39.

\subsubsection{Laminar airflow ventilation systems}

In order to obtain a ultraclean laminar airflow, laminar ventilation systems are used. These sort of ventilation systems are recommended for environments which require Class I style of surgeries. The category I style or class 1 style of surgeries involves orthopaedic, transplant, and other specialized surgeries. Laminar airflow ventilation systems also are used for isolation rooms, those who met with fire accidents and also in laboratories. In order to satisfy the category I type surgery characteristics, these ventilation systems are combined with the highefficiency particulate air filters (HEPA), along with an occasional and uniform velocity. If laminar airflow ventilation systems are used alone it can divide the operating theatre in two zones. There are two styles of laminar airflow system, the horizontal and also the vertical. So as to beat the problems related to vertical airflow laminar airflow system the horizontal laminar airflow system was enlarged. However, the horizontal supply of air in operating rooms generally is disrupted by the surgical team (Dharan et.al, 2002)17.

In operating rooms comparing to the horizontal, the vertical laminar airflow system is more successful, because, and also more useful in accordance with some studies. In accordance with literature review and studies made by (Friberg, 1999)21, the ultra clean air system ensures the potential of protection for the patient. Based on the look of the diffuser the surgery are often divided into one zone or in two zones by laminar airflow systems. As an example, a full ceiling diffuser defines only one zone. But within the case of small diffuser it divides the operating theatre in two zones. (Mermarzadeh et,al ,2002)38 made a comparative study of operating suite ventilation systems. They evaluated the laminar airflow, unidirectional, conventional and non aspirating and displacement diffuser types so as of contaminant deposition on an operating room and backtable. Various specifications like volume flow for every ventilating system are shown through these ventilation systems. The most aim of this study is to judge various characteristics, such as, the ventilation rate of flow effects, diffuser type and placement, supply temperature and exhaust location. The methodology used was numerical simulation. 
This research study had important analysis in terms of infection control. The obtained results shown that the laminar airflow ventilation systems are better in surgery with some care and also it ends up in better ventilation effectiveness.

(Buchana Dunn-Rankin,1998)6, studied two ventilation systems: cross flow and "impinging-flow". The cross-flow presented airflow moved horizontally over the table, and also the particles were lifted toward the ceiling by strong air currents and so carried to the outlet and removed. During this case the heating source had a touch effect within the airflow. The impinging flow was opposed by rising natural convection currents caused by the heating loads that prevented the inlet air from properly ventilating the surgical site and its distribution within the room. Cross-flow was more practical within the removal of contaminant than impinging flow. The results showed that surgical team, patient and lights had great effect on the airflow because of heat loads, which had a major influence within the transport of air contaminants. Other papers also reported these effects in laminar airflow, unidirectional and plenum ventilation systems, mainly which present vertical air supply. (Melhado et.al, 2006)39.

(Wanner et.al., 1980)56, evaluated operating rooms equipped with a "modern" ("Greenhouse") laminar airflow and with so called "germ-stop wall". The second system consisted of glass wall with a gap to maneuver within the patient, and which divided the hospital room in two zones. The primary zone is cleaner than secondary zone respectively, said the surgical team, anesthesiologist and other support staff. Up to the mark of bacteria levels the second system reported good results. (Melhado et.al, 2006)39.

The laminar airflow also had great impact on postoperative infection rate, when combined with other ventilation systems, for instance, the helmet aspirator system. Some studies reported a discount, not significant, to the count of aerobic airborne and sedimenting bacteria-carrying particles within the operating theater employing a mobile laminar airflow screen additionally to traditional (mixing) ventilation. These same levels were achieved with a laminar airflow with HEPA filters. This subject has to be investigated in details, because some studies presented different comments for a same style of ventilation for the identical kinds of surgeries. (Melhado et.al, 2006)39.

\subsubsection{Combined ventilation systems}

Various types of ventilation systems were evaluated and compared in the study of (Friberg et.al., 1996)23. The ventilation systems were: "Floormaster" with the supply of air from the floor and exhaust through the ceiling, and the Plenum with the diffuser of supply air inclined and in a wall, while the exhaust at floor level. The conclusions were "that an upward displacement system will lead to increased counts of airborne and sedimenting bacteria and thus increase the risk of postoperative infection in comparison with convention operating room ventilation systems". Two types of ventilation systems used in Brazil and in other countries are the Unidirectional ventilation system in addition the Linear System. These combined systems define three zones in the operating room-Zone 1 (ultra clean) stay the patient, Zone 2 the surgeon, auxiliary nurse and instrument tables, and Zone 3 the anesthesiologist, the circulating nurse and other support staff. The Linear system here "works" like a barrier, dividing the Zone 2 from Zone 3. This barrier does not permit that the less clean air enter in the Zone 2, therefore ensuring satisfactory protection for the instrument table. Hospitals that used these systems in operating room have reported lower infection rate. Reporting the results of operating room, it was observed that high complexity was involved in this subject, and that it is very difficult to control some variables. In different types of surgery it was observed different layouts. However, in the hip surgeries (zone I and III) the layout of table instruments, position of the operating table, light and people were similar. In all surgeries the instrument table had out if the zone 1, or in some case between zone 1 and zone 2 . In all surgeries the anesthesiologist, anesthesiologist residents, nurses and other people stay in Zone 2.. The "mayo" table usually was in Zone 1. However, in the cases that the surgeon need more space because the type of surgery, or when it was used the X-Ray, this table was kept between Zone 1 and Zone 2, or totally in the Zone 2. All equipment was kept in the limit Zone 1 in the majority of the surgeries. The position of these can result in disturbed airflow dynamic of the supply air). The "trashes" always were located closer to the instrument table. The "trash", closer from the doors, and always it was in this corner and covering the exhaust. The position of the people and instrument table changed in accordance with the type of surgery (Melhado et.al, 2006)39.

\subsection{Ultraviolet air recirculation systems}

Surgical Infection Society (SIS) introduced a air recirculation/filtration system for a cosmetic surgery at an outpatient surgery center. The recirculation unit utilizes a hybrid of biological and physical systems to get rid of bacteria, fungi and viruses from the air. Its key biocidal technology may be a solid state germicidal irradiation 
system which provides simultaneous physical filtration and irradiation of high volume air flow. The system utilizes C-band ultraviolet radiation (UV-C) at a $254 \mathrm{~nm}$ wavelength diffused into a solid media which is gas and radiation permeable. While organisms are slowed or trapped within the solid media, they're inactivated by the interior UV-C dosage. This has the effect of accelerating the inactivation efficiency over prior UV technologies. The HUAIRS device is effective in reducing airborne contamination and improving air quality within the operating room, supporting reduced SSI (Surgical site infection) risk for patients and improved safety for the surgical team. The look of a UVC system must be optimized to maximize dosage while maintaining adequate air flow volumes. At a given UVC radiation output and distance, air flow volume and UVC dosage received are inversely related, leading to necessary compromise within the design of such systems. The crystalline UVC (C-UVC) system was developed to maximize UVC dosage and inactivation efficiency while maintaining high air flow volumes. The C-UVC device may be a novel in-room air disinfection-recirculation unit. It utilizes a hybrid of biological and physical systems to get rid of bacteria, fungi and viruses from the air. Its key biocidal technology could be a reactor system which provides simultaneous physical filtration and irradiation of high-volume air flow with minimal resistance. The reactor system utilizes C-band UV (UVC) focused on a reaction chamber stuffed with a mess of clear cylindrical silicate crystals. The silicate crystals function as a solid media filter, slowing and trapping organisms as they pass via the air stream. a novel feature of silicate crystal is that it may be efficiently penetrated by UVC irradiation. Therefore, while organisms are slowed or trapped within the solid crystalline matrix, they're inactivated by the penetrating UVC dosage. This has the effect of skyrocketing the inactivation efficiency over prior UV technologies (David Kirschman et.al, 2014)20. A image of the crystalline UVC (C-UVC) air disinfection-recirculation device is shown within the figure 5.

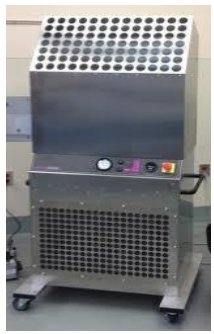

Figure 5. Crystalline UVC (C-UVC) air disinfection-recirculation device

\subsection{Air cleaning technology}

Air cleaning technology is one of the methods to reduce airborne contaminants concentration by delivering air through a high-efficiency air filter either through turbulent flow or laminar flow based on the distribution of air (Bingli Zhang etal 2020)9.

\subsection{GSS (glass flow stabiliser)}

As the name suggests glass material is preferred here to control the airborne microbial contamination. Here aflow stabilizer made of good quality compound laminated glass is present. It make the protective zone area big by (The protective zone is formed by way of a unidirectional flow. It covers the overall sterile environment for the surgical treatment. The sterile environment also includes the fabric and instrument table and also the persons in sterile clothing. As a result, the patient, surgical staff, material and instruments are optimally protected against airborne particles and bacteria. The area of the protective zone is marked on the ground) preventing the constriction of the laminar air flow under the outlet. As a result, the airborne particles from outside can't enter into the protective zone of the glass pane. The longer the glass pane is, the larger the protective zone is. When planning, it must be ensured that no collision risk with medical installations (weisstechnik, 2017)58.

\subsection{SSV (Surgical smoke ventilation) extraction of surgical smoke}

Surgical smoke (Surgical smoke is the gaseous by-product produced when tissue is dissected or cauterized by heat generating devices such as lasers, electrosurgical units, ultrasonic devices, and high speed burrs, drills and saws. Among these devices, the most common sources are electro cautery ablation and laser ablation) which occurs in surgery due to laser and ultrasound instruments, high frequency, radio frequency. The harmful smoke gases and aerosols rise upward and also the surgical staff inhales them. Surgical masks and mobile extraction devices have proven to be less practical during this regard. The surgical smoke extraction sucks the smoke from where it's formed (ie) right on the wound. In doing so, the extraction tube is positioned by the surgeon. By doing this an optimum extraction effect with a transparent view are often achieved. The air which is extracted is goes upwards via a tube and is transported though a pump or a fan to the exhaust. This method significantly reduces 
smoke particles and unsightly odors. Furthermore, regular filter changes aren't necessary unlike with mobile devices. The location of the pump or fan outside of the operating room avoids sound pollution. Together with an unidirectional flow canopy, the innovative smoke extraction offers the simplest efficiency and so optimum protection additionally to comfortable working conditions for the surgical staff. Alternatively, the extraction may be coupled to the medical instrument and may be automatically switched on and off (weisstechnik, 2017)58.

\subsection{Continuous particle monitoring system (CPM)}

The CPM system needs more hygiene requirements within the operating suites and helps to effectively protect patients from airborne particles and bacterial burden. Controlling the air quality can provide a very important aid in avoiding nosocomial infections through contaminated surgical instruments. Through this way, it serves to confirm quality. Furthermore, it allows the precise documentation of the clean air quality. The air is sucked in through a air pump via a tube right within the critical area above the surgical instruments or the instrument table, and also the air is conducted through a particle counter that continually measures the air quality. If the pollution increases, e.g. through the vigorous movement of the surgical staff, then the clean air supply is additionally automatically increased for the amount of the increased burden. A screen or a light-weight shows the present status of the air quality with its corresponding time (weisstechnik, 2017)58.

\subsection{ACS (Air curtain system)}

The most effective style of operating suite mechanical system is the air curtain system. Air curtain systems accommodates streamline flow diffusers, and a fringe of high-velocity air curtains combined with sidewall returns located near the ground. This mix allows for slow-moving, clean air to be vertically supplied from the streamline flow diffusers, while the air curtains along the perimeter help to make a barrier against contaminants. Air curtain systems offer many benefits compared to streamline flow systems. In general, an air curtain system can allow careful control of velocities and particles, however, the Metalaire Periflow system goes a step further and combines accurate airflow balancing with an easy installation.

The air curtain system has several distinct advantages over the streamline flow system utilized in an surgery. First, with the peri flow type system, the air curtains help to keep up low velocity streamline flow over the table. An issue with streamline flow diffusers is that airflows from adjacent units tend to coalesce and mix, which ends up in an increased velocity, and also the entrainment of particles.

However, on the contrary, when air curtains are utilized in conjunction with streamline flow diffusers, the high velocity air curtain creates a jet with lower pressure which induces the laminar airflow. The central laminar airflow is pulled outward into the perimeter high-velocity jet, filling the zone within the curtain with lowvelocity laminar airflow. This induction and expansion maintains true streamline flow within the operating zone and avoids the problems one would encounter using streamline flow units alone. Since the low sidewall returns regularly extract the airflow, this helps to stay particulates flowing far from the operating room table and out of the space (Mike Collins ,2018)41.

The air curtain system is an innovative flow stabilizer - complete and screenless. It is ideally suited to operating theatres, during which there's an increased risk of collision when employing a stationary glass flow stabilizer to a mess of medical installations (e.g. operation lamps, mobile angiographic units or canopy-mounted supply units). Within the air curtain systems, the air flow is stabilized by air rather than a glass pane. The air is guided via the frame profile and duct tracks that are integrated around within the frame profile. The air volume is regulated very easily (Mike Collins,2018)41.

Another important function of an air curtain system is to stop any particles from outside the curtain from reentering the sterile field. The air curtain system effectively creates a fence against external contaminants from outside the operating area. Since the streamline flow diffusers provide sterile air over the surgery table, it's important to make sure that no contaminants can make it to the operating area. An air curtain perimeter accomplishes this by establishing a boundary by which no particles can infiltrate from outside the table area. The sterile supply air washes and take away particulates over the patient and bio hazardous materials which are then hyped up by the high velocity jet and extracted by the sidewall return (Mike Collins, 2018)41.

The Metalaire peri flow system has its own individuality because the entire perimeter of air curtains consists of a single plenum. Only two duct connections are permitted for the entire perimeter through this single "loop" plenum. This helps the installer to save much time and effort, since many comparable systems use a handful of 
inlet connections. When a system uses multiple inlets, each inlet will require airflow balancing to maintain equivalent velocities through each air curtain and any change in overall system pressure will require rebalancing. If the air curtains have different velocities, this can cause the entire system to function incorrectly thereby increasing the chance of contamination (Mike Collins ,2018)41.

In the Peri flow system, once both duct connections are active, the complete loop becomes pressurized. Since the plenum loop becomes pressurized, each air curtain along the perimeter will maintain the identical velocity. This makes the Metalaire Periflow system one amongst the best operating room systems to put in, likewise united of the foremost effective systems for maintaining a clean operating suite (Mike Collins, 2018)41.

\subsection{Scavenging equipment}

Scavenging is the collection and removal of vented anesthetic gases from the operating room. Since the amount of anesthetic gas supplied usually far exceeds the amount necessary for the patient, operating room pollution is decreased by scavenging equipment. The scavenging equipment consist of gas collection assembly, (tubes connected to APL valve (adjustable pressure limiting valve) and vent relief valve), transfer tubing, scavenging interface, gas disposal tubing (to carry gas from interface to disposal assembly), gas disposal assembly (active or passive - active most common, uses the hospital suction system). Scavenging may be active (suction applied) or passive (waste gases proceed passively down corrugated tubing through the room ventilation exhaust grill of the operating room) (Michael et.al, 2019)43. Active systems require a means to protect the patient's airway from the application of suction, or buildup of positive pressure. Passive systems require that the patient be protected from positive pressure buildup only. If a fresh gas flow-sized volume enters the breathing circuit each minute, the same flow must leave it, or barotrauma (injury caused by a change in air pressure, affecting typically the ear or the lung) will result. Scavenger and operating room ventilation efficiency are the two most important factors in reduction of waste anesthetic gases. A significant reduction in operatingroom pollution was obtained by use of easy scavenging equipment. Scavenging of anesthetic vapors outside the operating room led to a 97.3 per cent reduction of overall mean concentration of halothane within the hospital room atmosphere and reduction of 72 per cent in end tidal samples of anesthetists, with the Magill semi closed circuit (Novair,2018)45. Anesthesia machines and breathing systems delivering halogenated hydrocarbon anesthetics and/or inhalation anesthetic mustn't be operated unless they're equipped with a functional scavenging system. An efficient scavenging system is capable of reducing ambient concentrations of waste gases by up to 90\%. To be effective, a scavenging system, it should not leak and must control the concentration of trace anesthetics in ambient air (Kumar, 2015)31.

\subsection{Positive pressure rooms}

Positive pressure rooms maintain a better pressure inside the treated area than that of the encompassing environment. This suggests air can leave the area without circulating back in. During this way, any airborne particle that originates within the room are filtered out. Germs, particles, and other potential contaminants within the surrounding environment won't enter the area. In medical settings, a positive pressure room (protective environment) allows staff to stay vulnerable patients safe from infections and disease. The aim of positive pressure is to make sure that airborne pathogens don't contaminate the patient or supplies in this room. As an example, positive pressure may well be employed in an operating room to guard the patient and sterile medical and surgical supplies. Positively pressured rooms are typically considered the cleanest rooms within the hospital (Air innovations, 2020)5.

Transmission of airborne particles within the operation theaters is sometimes prevented by the employment of a positively pressurized operating rooms. The intentional over-pressurization air to effuse of the operating room when doors open, which prevents adjacent air from penetrating into the operating room . However, the pressurization systems can become overwhelmed when doors open too over and over in quick succession or stay open too long. When the door is opened, the operating room mechanical system fails to keep up the specified positive pressure difference within the area, and leads to contaminant dispersion to the surgical zone. Door opening ends up in a failure in isolation conditions and leads to penetration of contaminated air from the adjacent area into the operating room (Air innovations, 2020)5.

\subsection{HEPA filters (High efficiency particulate air matter filters)}

The High efficiency particulate air (HEPA) filter provides the best protection from infection and contamination from airborne microorganisms, especially when engineered so that the filter is mounted, commonly at 
ceilings referred to as ceiling mounted HEPA filters or modules (Danforth, 2016)19. These filters have a long history of providing protection against airborne diseases. HEPA filters consist of a fiber glass of diameters between 0.5 and 2.0 microns in which airborne microbes and particles gets trapped. Ceiling mounted HEPA modules deliver clean, filtered air in a flow to the room that provide a sweeping effect that pushes contaminants out via the return grilles that are usually mounted on the lower wall. While there is no true unidirectional airflow, this top to bottom flow closely duplicates a "piston-effect." With ceiling mounted HEPA units, the air is as close to unidirectional flow in operating rooms as is feasible, based on the various types of equipment and instruments that typically interrupt unidirectional airflow patterns (Danforth, 2016)19.

HEPA filter module is shown in the figure 6.

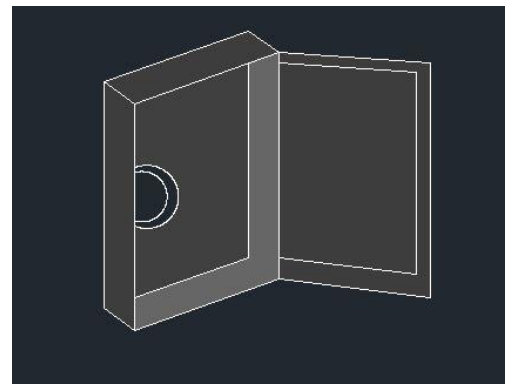

Figure 6. Ceiling mounted HEPA filter module

\subsection{Temperature and humidity control}

The level of temperature and humidity should be maintained at $21^{\circ} \mathrm{C} \pm 3^{\circ} \mathrm{C}$ (except for joints replacement where it should be $180^{\circ} \mathrm{C} \pm 20^{\circ} \mathrm{C}$ ) along with respective relative humidity between 20 to $60 \%$, though the ideal $\mathrm{RH}$ ( relative humidity) is considered to be $55 \%$. Appropriate devices to monitor and display these conditions inside the operation theaters may be installed (Revised guidelines for air conditioning in operation suites 2018)

\subsection{Temperature controlled air flow system}

A ventilation scheme, called temperature-controlled airflow (TAF), has recently been developed to reduce air pollution in operating room use. In contrast to the conventional mixing and laminar airflow ventilation, temperature airflow ventilation supplies air at various temperature levels and controls the air movement based on the temperature gradient. Specifically, cool air is discharged into the operating zone from ceiling mounted central diffusers to displace the contaminated air, whereas warm air is dispersed into the boundary of the operating room through surrounding diffusers to further dilute the airborne biological contamination of particles concentration .The central supply air is kept about $1.5-3^{\circ} \mathrm{C}$ cooler than the ambient air in the operating room and thus the incoming air is accelerated by gravity due to density difference and falls down to the surgical zone. The downward air streams are expected to overcome the convective currents developed from the heat sources - that is, the surgical staff, surgical equipment and lamps - and wash away biological contamination of particles from the patient and sterile instruments. The downward air velocity, dictated by the temperature difference between the incoming air and the ambient room air, should be sufficient for the clean air to reach the surgical site and provide the adequate washing effect. Hence, terminal airflow can be seemed as a combination of features of conventional mixing ventilation that dilutes the microbiological contamination and unidirectional airflow ventilation that directly removes the biological contamination of particles. The terminal airflow ventilation system has so far been installed in several hospitals in Sweden, Germany, Switzerland, the Netherlands and the United States. Based upon the information from Swedish hospitals, the installation cost for the terminal airflow system is higher than for conventional mixing ventilation systems (Cong wang, 2019)15.

\subsection{UVGI applications and solutions}

Ultraviolet germicidal irradiation (UVGI) works by degrading and deactivating bacteria mold spores, fungi and viruses (Carrier 2020)12. UVGI may be a proven technology, which was first used for disinfecting surfaces in 1877 and for the aim of air purification in 1935, which has the control of the transmission mechanism of tuberculosis. Hospitals have used UVGI for years to scale back the spread of superbugs (Superbugs are strains of bacteria, viruses, parasites and fungi that are proof against most of the antibiotics and other medications commonly accustomed to treat the infections they cause) and to disinfect surgical suites (Carrier 2020)12.Ultraviolet-C (UV-C) refers to the short-wavelength (200-280 nanometers) energy employed in UVGI applications. The everyday source of artificial ultraviolet disinfection could be a low-pressure mercury vapor 
lamp that gives energy at approximately 254 nanometers UV, near the foremost effective germicidal wavelengths. Current research into far UV ( 222 nanometers) looks promising for pathogen inactivation with less amount of risk to humans. UVGI solutions are widely deployed within the HVAC (Heating, ventilation, air conditioning) industry to eradicate the mold and fungus that accumulate on wet surfaces of HVAC units like the evaporator coil and drain pan. This method called as "bio film source control," focuses UV-C energy on these static contaminated surfaces, successfully eliminating the objectionable "dirty sock" odor that when plagued HVAC systems. We all know that a clean coil has lower pressure drop than a biofilm-clogged coil, thereby preserving original performance. Used consistently in an HVAC system, non-ozone producing UV-C lamps selected to supply maximum dosage at 45 to 55 degrees $\mathrm{F}$ ( 7 to 13 degrees $\mathrm{C}$ ) in a very rapidly moving airstream - will ultimately destroy organisms on the coil and condensate pan. This process can return these components to an "as installed" condition and virtually eliminate the necessity for coil cleaning. With the increase of COVID-19, UVGI applications have taken on a replacement and immediate role in protecting and improving the workplace. The Centers for Disease Control (CDC) guidelines conclude that, "as a supplemental air-cleaning solution, UVGI is effective in reducing the transmission of airborne bacterial and viral infections in hospitals, housing and in classrooms. Introduced within the HVAC systems of economic buildings alongside increased outdoor air ventilation, proper humidity controls and upgraded filtration technologies, UVGI solutions can provide an efficient technology within the war against the SARS-CoV-2(Severe acute respiratory syndrome corona virus 2) ( Carrier, 2020)12.

UVGI offers a spread of potential disinfectant strategies for the built environment. In-room irradiation of unoccupied spaces by autonomous robots armed with an array of UV-C lights is being deployed in patient rooms and operating theaters in Europe, China and North America. Standing nearly 6 feet tall and equipped with lidar sensors, these robots follow a digital map, traveling to and from their charging stations (including up and down elevators) to disinfect rooms in about quarter-hour. For safety, they're programmed to prevent immediately if they detect motion (Carrier, 2020)12.

\subsection{Individual air supply systems}

Individual air supply system with a fan will improve indoor air quality. Switching on the device causes a very significant change. Here lower natural ventilation ducts sections, were used as air supply channels to bring air inside the room. Exhaust grilles were located in the adjacent rooms. As a result of this the carbon dioxide concentration was reduced in the indoor air quality. An air quality indicator was used to measure the carbon dioxide concentration. A large carbon dioxide concentration reduction has been confirmed by rigorous statistical analysis (E. Zender-S'wiercz, 2017 )60.

\subsection{Operation suite re circulating air canopy}

The optimized low-turbulence re circulating air canopy consists of an air outlet element, terminal airborne particle filters, a plenum with sound absorbers and re circulating-air modules. So as to ensure maximum safety alongside optimum efficiency, the ultra laminar airflow (ULA) mixes the return air and provide air within the re circulating-air module. For this purpose, the theatre air is sucked into the re circulating-air module and mixed with the availability air coming from the air-conditioning unit. The mixed air is transported to the plenum positioned above the filter. From there, it's conducted in its particle filtered state as clean air into the operating room and therefore the preparation area, where it forms a protective zone (Weisstechnik, 2017)58.

\subsection{Air sampling methods}

Two types of methods are used for microbiological sampling of air in operating rooms (ie) active air sampling method and passive air sampling method. In active air sampling, the sampling instrument (e.g. a slit sampler) actively pulls in a pre-defined volume of air through or over a collection device such as a filter or an agar plate. The forming units per unit volume of air (i.e. CFU/m3) represents the microorganisms collected by the collection device where it will be incubated and the level of contamination is expressed as colony.

At the same time, passive air sampling requires the use of a settle plate (e.g. a Petri dish with a culture medium) which is exposed to the air for a certain period of time. The microbiological particles which eventually sediment out of the air and settle over the surface of the plate over a period of time exposure are subsequently incubated. The microorganisms on the settle plate are counted and by relating both the plate area and exposure time and the level of contamination can be presented in the form of CFU/m2 /h (Cong wang, 2019)15. 
The passive air sampling is more efficient in sampling larger, faster-settling particles, as larger particles are more likely to sediment than are smaller particles (B. Friberg etal 1999)21. Considering the size distribution of biological contamination of particles, the passive sampler needs to work continuously over a long time period to collect a sufficient amount of microorganisms. In contrast, the active sampler samples air over a short time period can collect the microbes and thus can reflect the transient effect of individual events on the air quality in operating rooms, such as door openings and foot traffics in operating rooms. However, the passive air sampling is more convenient in practice than the active air sampling, as the settle plate is easily available and can be used without additional devices.

A study was made to determine the microbiological air quality in public buildings of Egypt such as a schools, libraries etc. Airborne bacteria and fungi were collected using Andersen two-stage sampler, where it divides particles into coarse $(\geq 7 \mu \mathrm{m})$ and fine $(<2.5 \mu \mathrm{m})$ size ranges. In this study particulate matter positively affected bacterial culturability and temperature negatively affected bacterial and fungal curturability in outdoor environment (Abdel Hameed Awada et.al, 2020)4.

The international standard (ISO19468-1) describes the available air sampling techniques but leaves the choice of method open. The best method to assess the microbiological contamination of air remains debatable and studies in this field are controversial. Some researchers recommend the use of passive air sampling, arguing that the surface count of bacteria is clinically more relevant to the risk of SSIs than the air count as the airborne contaminants that fall onto a critical surface is more harmful. (Napoli et.al 2012)47 suggested that both methods could be useful for general monitoring of air contamination but the choice should be made depending on the specific information to be obtained.

\subsection{Fan mounted surgical lamps}

An innovative design of the surgical lamp - fan-mounted lamp, is assessed and compared with a traditional closed-shape lamp. The performance of the 2 designs of lamps is evaluated under two ventilation schemes: laminar air flow and mixing ventilation. The operating room within the New Karolinska Solna University hospital is adopted due to the physical case where the 2 lamps have an analogous overall dimension. Both have a hexagonal cross-section with an outer diameter of $0.7 \mathrm{~m}$ and thickness of 0.15 m.A $0.2 \quad \mathrm{~m}$ diameter fan is installed inside the fan-mounted lamp, which creates a continuing pressure jump of $0.6 \mathrm{~Pa}$. The fan-mounted lamp resolves the matter that the traditional lamp has caused within the laminar airflow ventilation. When using the closed-shape lamp, laminar airflow performs even worse than the blending ventilation, leading to airborne biological contamination of particle concentration (BCP) $63 \mathrm{CFU} / \mathrm{m} 3$. Such a high level of biological contamination of particles concentration poses a heavy threat to patient safety in infection-prone surgery. Against this, zero contamination is achieved in laminar airflow ventilation (LAF) with the fan-mounted lamp. It's noteworthy that by using the fan mounted lamp the contamination level within the mixing ventilation can be reduced.

The closed shape lamp blocked the unidirectional airflow and leads to a stagnant area under the lamp. Thus, the washing effect expected in laminar airflow ventilation is severely weakened. The stagnant area is eliminated by the fan-mounted lamp that blows air to the table. Within the mixing ventilation, the air blown from the fanmounted lamp may help to wash the biological contamination of particles far away from the surgical site.

Therefore, the fan-mounted lamp improves the performance of both LAF and mixing ventilation. Quantitatively, applying the fan mounted lamp decreases the contamination level by nearly $100 \%$ within the LAF ventilation for both surface and airborne contamination. Within the mixing ventilation, the surface contamination decreases by $26 \%$ and also the airborne contamination by $41 \%$ when using the fan-mounted lamp. Hence, the fan-mounted lamp proves to be more practical within the LAF ventilation than within the mixing ventilation (Cong wang, 2019)15.

\subsection{Indoor Air Quality Sensors}

Sensors are devices which helps to detect some kind of input within the physical environment, for example, light, motion, temperature, etc. and transmit that information into a symptom which will be measured and transmitted electronically. During this case, an internal air quality sensor would detect the presence of a selected pollutant. Different kinds of sensors measure various things. Advanced technology has brought down the price of indoor air quality testing by creating smaller, less costly sensors. It is essential to have enough space to position them strategically throughout the building to supply reliable results. (The EPA recommends 
one sensor for each 10,000 square feet.). Core categories for sensor measuring are volatile organic compounds, moisture, formaldehyde radon. Sensors can able to measure methane, bio aerosols, and more. Sensors can be placed anywhere in the building especially near personnel heaters, floor fans and common areas such as staircase but it should be kept out of direct sunlight. A wall mount is best, as floors and ceilings might not be indicative of the particular environment. When mounting a wall sensor, consider the peak at which they must be placed. Most often typically they're mounted at height-level3 to 5 feet ( 0.9 to 1.5 meters) or slightly lower, if people primarily remain seated therein particular area. However, the relative molecular mass of some substances makes them gravitate toward the ground or ceiling. Based on what pollutant we are trying to trace we have to place the sensor accordingly. As an example, , methane is lighter than air so the very best concentrations inside buildings are usually near the ceiling. Conversely, ozone is heavier than air and in such case sensor remains near the ground. Not all sensors work the identical way;. If we are working with an online of things company like IOT (Internet of things), our indoor air quality monitoring equipment will send the sensor data to the cloud, where we can access it via software. We get immediate, real-time access to the monitoring results through a dashboard, which turns the information into visual charts that are easy to decipher. As we continue monitoring over time, the information we receive will paint an accurate picture of our building's overall air quality Once we understand that, we can have the data that we wish to maneuver forward. Sensors are the smallest amount expensive component of indoor air quality testing. The price of internet of things (IOT) sensors has dropped steadily over the last several years, and is probably going to still go down. The variability of the price related to this element has got to do with the amount of sensors that we would like to adequately cover our particular building. We don't need plenty of sensors; only thing is it's more important to position your sensors at the right place (IOTA, 2020)26.

A study was made for the estimation and forecasting purpose of indoor air quality for class rooms using low cost sensor. A single sensor was kept in a class room and by using multi layer preceptron (MLP) and extreme gradient boosting regression (EXGBR) the real-time estimation of the air quality was made in other rooms without placing sensors there. Accuracy of $95 \%$ and $96 \%$ were achieved during estimation and forecasting, respectively (Praveen Kumar Sharma et.al, 2020)49.

\subsection{Light emitting diode (LED) technology}

During a surgery in operating rooms, it is essential to make a patient painless. For this purpose anesthetic gases are used. Anesthetic gases are also called inhaled gases. Nitrous oxide, halothane, isoflurane, desflurane, sevoflurane are the anesthetic gases used in surgery for patients. When these anesthetic gases gets spread it may cause some issues such as malignant hyperthermia, postoperative nausea and vomiting (Derek T. Clar etal, 2020)18. So it is essential to monitor and control these anesthetic gases.

Lay-Ekuakille etal, 2013)36 has made a study in light emitting diode technology in order to monitor and control the anesthetic gases. Monitoring of gaseous compounds is essential in operating rooms. In this study an architecture of measurements was based on LED spectroscopy for monitoring operating rooms. It represented a control system based on pulse width modulation. Since the architecture is not a personal alert system and it is not based on chromatography, it could not monitor a large number of pollutants. But conversely, it would be able to measure with high accuracy a small number of chemical species included in pollutants.

\subsection{Natural Bio filters}

Bio filtration is a process in which a living organism is used to biologically degrade the pollutants. Bio filtration is an air pollution control technology which utilizes microorganisms to biologically degrade odors and other volatile air pollutants contained in waste air streams (Bioteg, 2020)10. In case of natural bio filters the roots of the plants are effective in the pollutants from the air, The soil present in the plants are washed off and it is planted in a soilless growth medium in a pot. Behind this entire system a rotating fan is kept through which pure air is circulated inside the room. By this method $57 \%$ methyl ethyl ketone present in the contaminated air is removed ( Jenni Luoma, 2018)29.

A short description of various methods which are discussed above is given in the table 1

Table 1. A short description of various methods which are discussed

\begin{tabular}{|l|l|l|}
\hline SNO & NAME OF THE METHOD & TECHNOLOGY APPLIED \\
\hline & & \\
\hline 1 & Increase in air supply & The air supply is increased in this method using a clean air system \\
\hline 2 & Ventilation systems & Supply of air is provided either through natural way or through \\
\hline
\end{tabular}




\begin{tabular}{|c|c|c|}
\hline & & mechanical systems in order to make the operation room air clean \\
\hline 3 & $\begin{array}{l}\text { Ultraviolet air recirculation } \\
\text { systems }\end{array}$ & $\begin{array}{l}\text { Ultra violet irradiation technology is used here along with a solid } \\
\text { media like crystal where the microbes such as fungi, bacteria gets } \\
\text { inactivated and gets trapped in solid media. }\end{array}$ \\
\hline 4 & Air cleaning technology & $\begin{array}{l}\text { Air filters with laminar or turbulent flow air supply are used here to } \\
\text { reduce the airborne contaminants and its infections here. }\end{array}$ \\
\hline 5 & Glass flow stabilizer & $\begin{array}{l}\text { In this method the entire area to be protected from the airborne } \\
\text { microbes in the operating room are covered with a glass plane so } \\
\text { that airborne microbes will not enter the protected area and cause } \\
\text { infections }\end{array}$ \\
\hline 6 & Surgical smoke extraction & $\begin{array}{l}\text { Surgical smoke that occurs during a surgery are extracted using } \\
\text { surgical extractors and the surgical smoke is taken via a tube to } \\
\text { outside the operating room. }\end{array}$ \\
\hline 7 & $\begin{array}{l}\text { Continuous particle } \\
\text { monitoring system (CPM) }\end{array}$ & $\begin{array}{l}\text { In this method airborne particles are continuously monitored in } \\
\text { order to ensure the good air quality of operating room. }\end{array}$ \\
\hline 8 & Air curtain system & $\begin{array}{l}\text { The air curtain system consist of streamline flow diffusers which } \\
\text { provides clean air and air curtains which acts as a barrier for } \\
\text { airborne contaminants }\end{array}$ \\
\hline 9 & Scavenging system & $\begin{array}{l}\text { In order to remove anesthetic gases from operating room } \\
\text { scavenging equipment is used. It consist of gas collecting tube } \\
\text { through which gas is collected and a interface for the gas storage } \\
\text { and disposal tube for the disposal of gas }\end{array}$ \\
\hline 10 & Positive pressure rooms & $\begin{array}{l}\text { In this method by maintaining a high pressure inside a operating } \\
\text { room the contamination of airborne microbes can be prevented. }\end{array}$ \\
\hline 11 & $\begin{array}{l}\text { HEPA filters ( High } \\
\text { efficiency particulate air } \\
\text { matter filters) }\end{array}$ & $\begin{array}{l}\text { A filter called HEPA filters are mounted on the ceiling. These } \\
\text { filters consist of a fiber glass to trap the airborne microbes. }\end{array}$ \\
\hline 12 & $\begin{array}{l}\text { Temperature and humidity } \\
\text { control }\end{array}$ & $\begin{array}{l}\text { By maintaining the level of temperature and humidity the airborne } \\
\text { microbial contamination is reduced in this method }\end{array}$ \\
\hline 13 & $\begin{array}{l}\text { Temperature controlled } \\
\text { airflow system }\end{array}$ & $\begin{array}{l}\text { In this method a cool air and a warm air are supplied from the } \\
\text { diffusers and these two mix up with the air present in the operating } \\
\text { room and dilutes the airborne particles }\end{array}$ \\
\hline 14 & $\begin{array}{l}\text { UVGI application and its } \\
\text { solution. Ultraviolet } \\
\text { germicidal irradiation }\end{array}$ & $\begin{array}{l}\text { In this method a low pressure mercury vapor lamp is used to } \\
\text { produce ultra violet irradiation with germicidal wavelength along } \\
\text { with HVAC systems in order to prevent the transmission of } \\
\text { airborne microbes and the infections caused by them }\end{array}$ \\
\hline 15 & Individual air supply system & $\begin{array}{l}\text { Here individual air supply system with a fan along with lower } \\
\text { ventilation ducts are provided to supply air in order to decrease the } \\
\text { level of carbon dioxide in operating room. }\end{array}$ \\
\hline 16 & $\begin{array}{l}\text { Operation theatre re } \\
\text { circulating air canopy }\end{array}$ & $\begin{array}{l}\text { Here air recirculation principle clean air is provided in the } \\
\text { operating room }\end{array}$ \\
\hline 17 & Air sampling methods & $\begin{array}{l}\text { In this method the airborne microbial contamination is evaluated by } \\
\text { the colony forming units of microbes. }\end{array}$ \\
\hline 18 & Fan mounted surgical lamps & $\begin{array}{l}\text { Here fan mounted surgical lamps along with laminar airflow } \\
\text { ventilation systems are used to reduce the airborne microbial } \\
\text { contamination }\end{array}$ \\
\hline 19 & Indoor air quality sensors & $\begin{array}{l}\text { Sensing devices called as sensors that are used to detect the air } \\
\text { pollutant in a operating room are used here. }\end{array}$ \\
\hline 20 & Light emitting diode & $\begin{array}{l}\text { It is a monitoring and control system. where anesthetic gases are } \\
\text { monitored and controlled in operating room. }\end{array}$ \\
\hline 21 & Natural Bio filters & $\begin{array}{l}\text { By using the roots of plants along with soilless growth medium the } \\
\text { pollutants from the air is removed. }\end{array}$ \\
\hline
\end{tabular}

\section{Conclusion}

From this review paper we can understand the impact of airborne microbes in operation suites and also various methods are discussed here to reduce the impact of airborne microbes in operation suites. According to the capacity of the operating room, no of persons working in the operating room, along with economic aspect effective methods can be chosen to reduce the air pollution in operation suites of hospitals. Along with 
these methods safety and precaution measures such as changing gloves, changing clothes, washing surgical instruments can also be done to control air contamination in the operation theaters.

\section{References}

1. Ankita Kankaria,et.al ,2014. Indoor air pollution in India: Implications on health and its control. Indian journal of community medicine, Volume 39 Issue 4 pp 203-207.

2. Alessandra Cincinellietal, 2017|. Indoor air quality and health .International journal of research and public health14(11), 1286.

3. Alastair Lewis ,2019. Air purification is catching on - but it may be doing more harm than good , The Conversation.

4. Abdel Hameed Awada et.al, 2020. Air microbial quality in certain public buildings, Egypt: A comparative study. Journal of Atmospheric Research.

5. M. Tholkapiyan, A.Mohan, Vijayan.D.S, A survey of recent studies on chlorophyll variation in Indian coastal waters, IOP Conf. Series: Materials Science and Engineering 993 (2020) 012041, 1-6.

6. Buchanan, et.al ,1998. Measurement and prediction of indoor air flow in a model room, Energy and Buildings 35(5): pp 515-526.

7. Bryan Heitzmann, 2015. Challenges, Considerations, and Concerns of indoor air quality. Pennsylvania Housing Research Centre.

8. Badia et.al J.M. ,2017. ,Impact of surgical site infection on healthcare costs and patient outcomes. A systematic review in six European countries, Journal of Hospital Infection. pp 1-15.

9. Gopalakrishnan, R., Mohan, A., Sankar, L. P., \& Vijayan, D. S. (2020). Characterisation On Toughness Property Of Self-Compacting Fibre Reinforced Concrete. In Journal of Environmental Protection and Ecology (Vol. 21, Issue 6, pp. 2153-2163).

10. Bioteg ,2020 .Bio filter/ odor control/ waste air treatment, newsletter.

11. Chow et.al ,2005. Ventilation Performance in Operating Theatres against Airborne Infection: Numerical Study on an Ultra-Clean System. Journal of Hospital Infection, Volume 59, pp 138-147.

12. Carrier ,2020. Shinning a spot light on HVAC ultraviolet technologies to fight disease transmissions, newsletter.

13. Chauveux, D., 2015.Preventing surgical-site infections. Measures other than antibiotics Orthopaedics \& Traumatology. Surgery \& Research Volume 101, Issue 1, Supplement, pp 77-83.

14. A.Mohan, V.Saravana Karthika, J. Ajith , Lenin dhal , M. Tholkapiyan , "Investigation on ultra high strength slurry infiltrated multiscale fibre reinforced concrete", Materials Today : Proceedings , Volume 22, 904-911, 2020.

15. Cong wang ,2019, Ventilation Performance in Operating Rooms: a Numerical Assessment KTH Architeture and the Built Environment.

16. Carmen Irniguez et.al . Contrasting patterns of temperature related mortality and hospitalization by cardiovascular and respiratory diseases in 52 Spanish cities. Environmental Research Journal.

17. Lavanya Prabha, S., Gopalakrishnan, M., Neelamegam, M., Development of high-strength nanocementitious composites using copper slag, ACI Materials Journal, 2020, 117(4), pp. 37-46.

18. 18-Derek T. Clar et.al,2020. Anesthetic Gases STAT PEARLS.NCBI.

19. Danforth , 2016, Operating room air filteration systems, newsletter.

20. David Kirschman et.al, 2014, Ultraviolet in-room air disinfection and recirculation greatly reduces viable airborne particle concentrations in the operating room setting.

21. Friberg et.al ,1999. Correlation between surface and air counts of particles carrying aerobic bacteria in operating rooms with turbulent ventilation, an experimental study. Journal of Hospital Infection. pp 61-68.

22. Friberg et.al ,2003. The addition of a mobile ultraclean exponential laminar airflow screen to conventional operating room ventilation reduces bacterial contamination to operating box levels. Journal of Hospital Infection. Vol. 55, Issue 2, pp 92-97.

23. Lavanya Prabha, S., Dattatreya, J.K., Neelamegam, M., , Investigation of bolted RPC plate under direct tension, Journal of Structural Engineering (Madras), 2009, 36(5), pp. 333-341.

24. Francesco Romano, 2020. Operating Theatre Ventilation Systems and Their Performance in Contamination Control. "At Rest” and "In Operation" Particle and Microbial Measurements Made in an Italian Large and Multi-Year Inspection Campaign. A article in International Journal of Public Health and Environment.

25. Gwo-Hwa Wanet.al. Long-term surveillance of air quality in medical center operating rooms. National library of medicine.

26. IOTA, $2020 \mathrm{https}: / / \mathrm{www}$.iotacommunications.com/blog-category/air-quality.

27. Jane E Brody,1981. Dangers of indoor air pollution, newsletter 
28. John Charnley, 1964 .A clean-air operating enclosure, $\underline{\text { Volume 51, Issue } 3}$

29. March 1964, pp 202-20.

30. Jenni Luoma , 2018, Naava and biofilteration- natural indoor air for everyone, newsletter.

31. Kappstein, 2001. Hygienic measures in the surgery department - What has been shown to be exaggerated?.Tagliche Praxis 42(3): pp 653-60.

32. Prabha, S.L., Surendar, M., Neelamegam, M., Experimental investigation of eco-friendly mortar using industrial wastes, Journal of Green Engineering, 2019, 9(4), pp. 626-637

33. Kasdekar., M.M., et.al,2016. Air Quality Monitoring of Operation Theaters in Government Medical College and Hospital, Aurangabad, India International Journal of Current Microbiology and Applied Sciences ISSN: 2319-7706 Volume 5 Number 6 pp. 42-49.

34. Louisvilleky,2020. Air pollution control district, a newsletter.

35. Lidwell et.al, 1980. Journal of Hospital InfectionVolume 1, Issue 4, December .pp 285-287.

36. Lidwell et.al , (1988). Air, antibiotics and sepsis in replacement joints. Journal of Hospital Infection .Volume 11, Supplement C. pp 18-40. 